\title{
SUI NUCLEI CHE SI RIPRODUCONO PER ITERAZIONE ${ }^{\text {}}$ ).
}

\author{
Nota di Ermenegildo Daniele (Catania).
}

Adunanza del, 9 novembre 1913 .

Fra $\mathrm{i}$ nuclei che furono oggetto di particolare studio compaiono in prima linea, per la loro semplicità ed importanza, quelli della forma

$$
\varphi_{1}(x) \psi_{1}(y)+\cdots+\varphi_{m}(x) \psi_{m}(y) \text {. }
$$

Se le $\varphi$ e le $\psi$ si suppongono costituire due sistemi di funzioni (reali) biortogonali, questi nuclei si possono caratterizzare dicendo che coincidono coi loro iterati, cioè soddisfano alla condizione

$$
K(x y)=\int_{a}^{b} K(x s) K(s y) d s .
$$

Possiamo ora domandarci più in generale: esistono dei nuclei $i$ quali si riproducano, non già dopo una sola, ma dopo $n$ iterazioni? Tali nuclei, come ora vedremo, esistono effettivamente, e si presentano come una immediata generalizzazione di quelli dianzi ricordati.

I. Chiamando, al solito, $K_{1}, K_{2}, \ldots$ i successivi iterati di $K(x y)$, si ha per de finizione dell'iterato d'ordine $n$ (convenendo di fare $K_{\mathrm{o}}=K$ ):

$$
K_{n}(x y)=\int_{a}^{b} K(x s) K_{n-1}(s y) d s .
$$

La questione che ci proponiamo è dunque la seguente: costruire un nucleo $K$, tale che sia

$$
K_{n}=K \text {. }
$$

La soluzione del problema è fornita dai noti teoremi generali, che insegnano a rappresentare un nucleo mediante le sue costanti caratteristiche.

I) Avevo già ricevuto le bozze di questa Nota, quando presi visione della Memoria postuma di G. LAURICELla: Sopra l'algebra delle funzioni permutabili di $2^{a}$ specie [Annali di Matematica pura ed applicata, serie III, tomo XXI (1913), pp. 317-351]. Il problema trattato in questa Memoria è, da un lato, molto più generale del mio, poichè l'equazione integrale di cui io trovo la soluzione è un caso particolarissimo di quella studiata dal LAURICELLA; d'altro lato il LAURICELla considera soltanto nuclei simmetrici, mentre io astraggo da questa limitazione.

Catania, 20 novembre 1913. 
Premetteremo questo lemma: Un nucleo soddisfacente alla (I) e privo di costanti caratteristiche eे necessariamente nullo.

Difatti dalla (I) segue immediatamente:

$$
K_{n+1}=K_{1}, \quad K_{n+2}=K_{2}, \ldots
$$

ed allora calcolando le traccie, cioè le costanti $t_{i}$ definite da

si trova pure:

$$
t_{i}=\int K_{i-1}(s s) d s,
$$

$$
t_{n+1}=t_{1}, \quad t_{n+2}=t_{2}, \ldots
$$

Si sa che condizione necessaria e sufficiente affinchè un nucleo non abbia costanti caratteristiche è che tutte le traccie, a partire dalla terza, sian nulle. Se dunque $K$, oltre a soddisfare alla (1), è pure supposto privo di costanti caratteristiche, segue dalle (3) che son nulle tutte le sue traccie; ed allora la funzione determinante $D(\lambda)$ si riduce ad $I$.

Calcoliamo anche la funzione intera $D(x y \lambda)$. Rappresentandone lo sviluppo in serie di potenze di $\lambda$ con

$$
D(x y \lambda)=A_{0}+A_{\mathrm{r}} \lambda+A_{2} \lambda^{2}+\cdots,
$$

si ha pei coefficienti $A_{i}$ (ricordando che tutte le traccie son nulle):

$$
A_{\mathrm{o}}=K, \quad A_{\mathrm{s}}=-K_{\mathrm{i}}, \ldots, A_{\mathrm{i}}=(-\mathrm{I})^{i} K_{i}, \ldots,
$$

e quindi, tenendo presenti le (2):

$D(x y \lambda)=\left\{K-K_{1} \lambda+K_{2} \lambda^{2}+\cdots+(-1)^{n-1} K_{n-1} \lambda^{n-1}\right\}\left(\mathrm{I}+\lambda^{n}+\lambda^{2 n}+\cdots\right)$.

La $D(x y \lambda)$ è dunque nient'altro che la funzione razionale in $\lambda$ :

$$
D(x y \lambda)=\frac{K-K_{1} \lambda+\cdots+(-1)^{n-1} K_{n-1} \lambda^{n-1}}{I-\lambda^{n}},
$$

e poichè s'è gì̀ trovato $D(\lambda)=\mathrm{r}$, così la funzione precedente ci darà pure il nucleo risolvente $K(x y \lambda)$. Il quale verrebbe cosl ad avere per lo meno un polo, cioe $K(x y)$ avrebbe almeno una costante caratteristica, contrariamente all'ipotesi fatta. Questa ipotesi non è dunque compatibile se non colla condizione $K=0$.

2. I nuclei da noi studiati sono cosi dotati di costanti caratteristiche. Sia $\lambda$ una di queste, e $\Phi(x)$ una corrispondente funzione fondamentale; sia cioè

$$
\Phi(x)=\lambda \int_{a}^{b} K(x s) \Phi(s) d s .
$$

E noto che $\lambda^{n+1}$ sard una costante caratteristica di $K_{n}(x y)$ con $\Phi(x)$ ancora funzione fondamentale, ossia si avrd, accanto alla precedente:

$$
\Phi(x)=\lambda^{n+1} \int_{a}^{b} K_{n}(x s) \Phi(s) d s .
$$

Ma se si suppone $K_{n}=K$, gli integrali dei secondi membri nelle (4) e (5) risultano identici, e quindi $\lambda$ deve verificare l'equazione:

$$
\lambda^{n+1}=\lambda,
$$


ossia, tralasciando la radice $\lambda=0$ che non puó essere una costante caratteristica:

$$
\lambda^{n}=\mathrm{r} \text {. }
$$

Per $\lambda$ non sono dunque possibili altri valori all'infuori delle radici $n^{\text {me }}$ dell'unità, che noi indicheremo $\operatorname{con} \varepsilon_{1}, \varepsilon_{2}, \ldots, \varepsilon_{n}$; in altre parole il nucleo $K(x y)$ ammette un numero finito di costanti caratteristiche, che sono le $\varepsilon_{1}, \ldots, \varepsilon_{n}$ od una parte di esse.

Allora noi siamo in grado di scrivere senz'altro nella sua forma più generale la parte di $K$ relativa ad una $\varepsilon_{i}$ qualunque. Questa parte si puó rappresentare con

$$
\frac{\sum_{i}^{m_{i}} p_{h}(x) \psi_{h}(y)}{\varepsilon_{i}}
$$

dove $\varphi_{1}, \ldots, \varphi_{m_{i}} ; \psi_{1}, \ldots, \psi_{m_{i}}$ sono due sistemi di funzioni biortogonali. Perciò $K$ dovrd essere della forma:

$$
K(x y)=\frac{\varphi_{1}(x) \psi_{1}(y)}{\alpha_{1}}+\cdots+\frac{\varphi_{\nu}(x) \psi_{\nu}(y)}{\alpha_{\nu}}+Q(x y)
$$

dove $: \alpha_{r}, \ldots, \alpha_{v}($ con $\nu \gg n)$ sono le radici $n^{\text {me }}$ dell'unita (ciascuna convenientemente ripetuta a seconda del rango della costante caratteristica ch'essa rappresenta); $\varphi_{1}, \ldots, \varphi_{\nu}$, $\psi_{1}, \ldots, \psi_{\nu}$ sono due sistemi di funzioni biortogonali (col che le parti relative alle diverse $\varepsilon_{i}$ sono ortogonali fra di loro); $Q(x y)$ è un nucleo privo di costanti caratteristiche, ortogonale a tutte le parti relative alle singole $\varepsilon_{i}$.

3. Viceversa, sia $K(x y)$ un nucleo costruito nel modo più generale secondo la (6) e conformemente alle condizioni enunciate per le $x$, le $p$, le $\psi$ e $Q$. Applicando a $K$ una prima iterazione, si trova:

e successivamente:

$$
K_{1}(x y)=\frac{\varphi_{1}(x) \psi_{1}(y)}{\alpha_{1}^{2}}+\cdots+\frac{\varphi_{v}(x) \psi_{v}(y)}{\alpha_{y}^{2}}+Q_{1}(x y)
$$

$$
\begin{aligned}
K_{2}(x y) & =\frac{\varphi_{1}(x) \psi_{1}(y)}{\alpha_{1}^{3}}+\cdots+\frac{\varphi_{\nu}(x) \psi_{\nu}(y)}{\alpha_{\nu}^{3}}+Q_{2}(x y), \\
K_{n-1}(x y) & =\frac{\varphi_{1}(x) \psi_{1}(y)}{\alpha_{1}^{n}}+\cdots+\frac{\varphi_{\nu}(x) \psi_{v}(y)}{\alpha_{\nu}^{n}}+Q_{n-1}(x y) \\
& =\varphi_{1}(x) \psi_{1}(y)+\cdots+\varphi_{\nu}(x) \psi_{\nu}(y)+Q_{n-1}(x y), \\
K_{n}(x y) & =\frac{\varphi_{1}(x) \psi_{1}(y)}{\alpha_{1}}+\cdots+\frac{\varphi_{\nu}(x) \psi_{\nu}(y)}{\alpha_{\nu}}+Q_{n}(x y) .
\end{aligned}
$$

Si ritrova quindi $K(x y)$ a condizione di fare $Q=Q$; e siccome $Q$ è privo di costanti caratteristiche, per il lemma del $\mathrm{n}^{\circ}$ I ciò significa prendere $Q=0$. Concludiamo: $i$ nuclei che si riproducono dopo $n$ iterazioni sono tutti e soli quelli della forma

$$
K(x y)=\frac{\varphi_{i}(x) \psi_{1}(y)}{\alpha_{1}}+\cdots+\frac{\varphi_{v}(x) \psi_{v}(y)}{\alpha_{v}}
$$


dove $x_{1}, \ldots, x_{v}(v \supseteq n)$ sono le radici $n^{m e}$ dell'unità, e le $\varphi_{1}, \ldots, \varphi_{v} ; \psi_{1}, \ldots, \psi_{v}$ sono due sistemi di fun-ioni biortogonali qualunque.

I successivi nuclei iterati di $K$ diventano poi:

$$
\begin{gathered}
K_{1}=\frac{\varphi_{1} \psi_{1}}{\alpha_{1}^{2}}+\cdots+\frac{\varphi_{v} \psi_{v}}{\alpha_{v}^{2}}, \\
K_{2}=\frac{\phi_{1} \psi_{1}}{\alpha_{1}^{3}}+\cdots+\frac{\phi_{v} \psi_{v}}{\alpha_{v}^{3}}, \\
\cdots \cdots+\varphi_{v} \cdots \\
K_{n-1}=\varphi_{1} \psi_{1}+\cdots+\varphi_{v} \psi_{v}
\end{gathered}
$$

hanno cioè ancora l'analoga forma di $K$, in quanto che una qualunque potenza di $\alpha_{i}$ è sempre una radice $n^{\text {ma }}$ dell'unità, per cui anche $K_{1}, K_{2}, \ldots$ avranno la proprietà di riprodursi dopo un certo numero di iterazioni: qui s'intende che le iterazioni di $K_{r}$ vanno fatte rispetto allo stesso $K_{r}$ come nucleo principale. In particolare si vede che $K_{n-1}$ è del tipo di quei nuclei che coincidono con tutti i loro iterati, cosa che si poteva dedurre direttamente osservando le (2) e la formola fondamentale

4. Casi particolari.

$$
\int K_{p}(x s) K_{q}(s y) d s=K_{p+q+\varepsilon}(x y) \text {. }
$$

a) Per $n=\mathrm{I}$ si ritrova il nucleo notissimo

$$
K=\sum_{i}^{v} \varphi_{i}(x) \psi_{i}(y)
$$

b) Per $n=2$ si ha:

$$
K=\sum_{i}^{\mu} \varphi_{i}(x) \psi_{i}(y)-\sum_{\mu+1}^{v} \varphi_{i}(x) \psi_{i}(y)
$$

che diventa, dopo una prima iterazione, della forma (8). La successione $K, K_{1}, K_{2}, \ldots$ è tale che tutti $\mathrm{i}$ termini di posto dispari sono uguali fra di loro, e cosi quelli di posto pari.

c) Nucleo simmetrico. - In questo caso nella (7) le $\varphi$ e le $\psi$ col medesimo indice sono uguali. Quanto alle $\varepsilon_{i}$ debbono essere reali, quindi fra le $\varepsilon_{i}$ non vi possono comparire che I ( $n$ dispari) oppure I e - I ( $n$ pari). Siamo cosi ridotti alle due forme corrispondenti ai casi $a$ ) e $b$ ), cioè

$$
\begin{aligned}
K & =\sum_{i}^{v} \varphi_{i}(x) \varphi_{i}(y), \\
K & =\sum_{i}^{\mu} \varphi_{i}(x) \varphi_{i}(y)-\sum_{\mu=1}^{v} \varphi_{i}(x) \varphi_{i}(y),
\end{aligned}
$$

dove le $\varphi_{i}, \ldots, \psi_{i v}$ formano un sistema di funzioni ortogonali. Un nucleo simmetrico, che si riproduca per iterazione, si riproduce dopo una o due itera ioni al piu.

La ricerca dei nuclei di quest'ultimo tipo si puó intendere contenuta implicitamente in una Nota di Lauricelda ${ }^{2}$ ), nella quale è risolto il problema di costruire un nucleo

2) G. Lauricella, Sopra i nuclei reiterati [Rendiconti della R. Accademia dei Lincei (Roma), vol. XX, $1^{0}$ semestre $19 \mathrm{II}$, pp. 885-896].

Rend. Circ. Malem. Palermo, t. XXXVII (10 sem. 1914). - Stampato il 28 novembre $\{113$. 
simmetrico di cui sia dato l'iterato d'ordine $n$. Per quanto l'equazione di Lauricella

$$
K_{n}=H(x y)
$$

ove $H$ è una funzione data, sia sostanzialmente diversa dalla $K_{n}=K$, nel caso della simmetria peró si puó facilmente, dalla soluzione del problema di Lauricella, dedurre quella del problema attuale.

Torino, 15 ottobre 1913 .

E. DANIELE. 